\title{
(2) OPEN ACCESS \\ PAX6 is frequently expressed in ependymal tumours and associated with prognostic relevant subgroups
}

\author{
Julian Tabasaran, ${ }^{1}$ Martin Schuhmann, ${ }^{2,3,4}$ Martin Ebinger, ${ }^{5}$ Jürgen Honegger, ${ }^{2,3}$ \\ Mirjam Renovanz, ${ }^{2,3,6}$ Jens Schittenhelm (1) 1,3
}

- Supplemental material is published online only. To view, please visit the journal online (http://dx.doi.org/10.1136/ jclinpath-2021-207526)

For numbered affiliations see end of article.

Correspondence to Professor Jens Schittenhelm, Department of Neuropathology, Eberhard Karls Universitat Tubingen, Tubingen, BadenWürttemberg, Germany; jens. schittenhelm@med.unituebingen.de

Received 4 March 2021 Accepted 20 May 2021

Check for updates

(c) Author(s) (or their employer(s)) 2021. Re-use permitted under CC BY-NC. No commercial re-use. See rights and permissions. Published by BMJ.

To cite: Tabasaran J, Schuhmann M, Ebinger M, et al. J Clin Pathol Epub ahead of print: [please include Day Month

Year]. doi:10.1136/

jclinpath-2021-207526

\section{ABSTRACT}

Aims An ependymoma shows divergent morphological and molecular features depending on their location. The paired box 6 (PAX6) transcription factor is a putative tumour suppressor and drives cancer cells towards a stem cell-like state. A transcriptome study reported high PAX6 expression in ependymal tumours, but data on protein expression are lacking.

Methods We, therefore, analysed PAX6 expression by immunohistochemistry in 172 ependymoma samples and correlated its expression to histology, WHO grade, anatomical location and molecular subgroups.

Results Mean PAX6 nuclear expression in ependymoma was $27.5 \%$ (95\% Cl 23.3 to 31.7). PAX6 expression in subependymoma (mean: $5 \%$ ) was significantly lower compared with myxopapillary (30\%), WHO grade II (26\%) and anaplastic ependymoma (35\%). Supratentorial ependymomas also displayed significant lower PAX6 levels (15\%) compared with spinal cord tumours (30\%). Expression levels in YAP1-fused ependymoma (41\%) were higher compared with RELassociated protein (RELA)-fusion positive tumours (17\%), while PAX6 expression was similar in posterior fossa group A (33\%) and B (29\%) ependymomas. KaplanMeier analysis in RELA-fusion positive ependymomas and posterior fossa group $B$ showed a significant better outcome for PAX6 at or above the cut-off of $19.45 \%$ compared with tumours with PAX6 below the cut-off. Conclusions We demonstrate that PAX6 is frequently expressed in human ependymal tumours and immunohistochemistry may be helpful in determining prognostic relevant subgroups.

\section{INTRODUCTION}

Ependymomas are central nervous system (CNS) tumours that predominantly arise in the lining cells of the ventricular system and spinal cord. Ependymal tumours are more frequent in children than in adults and depending on their tumour location they show divergent morphological, immunophenotypic and ultrastructural ependymal features. ${ }^{1}$ In adults, up to $46 \%$ of ependymomas are located in the spinal cord, while up to $90 \%$ of paediatric ependymoma are located intracranially. The current WHO classification recognises subependymoma grade I, myxopapillary ependymomas grade I, (classical) ependymoma grade II, anaplastic ependymoma III and a molecularly defined group, ependymoma, REL-associated protein (RELA)-fusion positive. ${ }^{2}$ Grade II and III tumours often do not correspond to their predicted prognosis, especially in different age groups and at different locations in the
CNS. ${ }^{34}$ Methylation profiling identified nine divergent molecular subgroups of ependymal tumours with tight relation to their anatomic sites and distinctive prognostic potential. ${ }^{5}$ Posterior fossa (PF) ependymomas can be distinguished by global levels of histone H3 K27-trimethylation into two clinical relevant groups designated PF A (PFA) and PF B (PFB). ${ }^{6}$ The molecular variant ependymoma, RELA fusion positive occurs supratentorial mostly in children and young adults and can be readily distinguished from other ependymomas by expression of L1CAM. ${ }^{7}$ RELA-like ependymomas carry ZFTA/C11orf95 fusions to MAML2/3, NCOA1/2 or CTNNA2. ${ }^{89}$ A smaller molecular group is seen in infants and characterised by predominantly YAP1 fusions. ${ }^{10}$ Spinal ependymomas may carry NF2 alterations or MYCN amplifications. ${ }^{11} 12$ Among the molecular groups, ependymoma group PFA, RELA-positive ependymomas and MYCN amplified spinal ependymomas carry an unfavourable prognosis. ${ }^{13}$ Recent consensus guidelines favour molecular classification over histological grading and suggested to assign WHO grade II to myxopapillary ependymoma because of frequent recurrences. ${ }^{13}$ Current standard of care include neurosurgical resection and radiotherapy. Most ependymomas are chemotherapy-resistant tumours and there is a demand to identify effective molecular targets for systemic therapies.

Paired box 6 (PAX6) is a transcription factor belonging to the highly conserved paired box family. The protein is important for the development of the CNS, eyes and endocrine pancreas ${ }^{14}$ and is expressed in precursors cells in developing human cerebellum. ${ }^{15}$ Dysregulation of PAX6 results in developmental disorders and tumour formation. ${ }^{16}$ PAX6 expression has been reported in non-small-cell lung cancer, breast, bladder, oesophageal and gastric cancer $^{17}$ and in several cancer cell lines including melanoma and haematological malignancies. ${ }^{18}$ PAX6 expression is highly tissue-dependent and in pancreatic cancer it is upregulated and results in oncogenic MET activation. ${ }^{19}$ In glioma cells, PAX6 acts as a tumour suppressor and inhibition of PAX6 resulted in increased proliferation and cell migration ${ }^{20}$ and in higher cell expansion under stressful conditions. ${ }^{21}$ The oncogenic role of PAX6 in brain tumours is not due to mutation in the PAX6 gene. ${ }^{22}$ Instead, PAX6 in glioma cells is a target of miR-223 and the miR-223/PAX6 axis regulates the growth, invasion and chemo resistance via PI3K/Akt signalling pathway. ${ }^{23}$ PAX6 gene expression from high-grade gliomas was reduced compared with adjacent normal brain and higher mRNA values 
were associated with better survival. ${ }^{20}$ Lastly, class I selective histone deacetylase inhibitor prodrugs such as Largazole pass the bloodbrain barrier and show upregulation of PAX6 in animal models indicating a potential treatment target. ${ }^{24} \mathrm{~A}$ study using oncogenic enhancer profiling showed that ependymoma tumour cells are enriched with PAX6 among other cancer-associated genes. ${ }^{25}$ Similarly a recent transcriptome study reported high PAX6 expression in ependymal tumours and animal experiments indicated that YAPMAMLD1 overexpression arises from PAX6-positive neural stem cells. ${ }^{26}$ Ependymal tumours also exhibit patterns of gene expression that recapitulate those of radial glia cells in the corresponding region of the CNS. ${ }^{27}$ Because PAX6 expression has been reported in radial glial cells of animal models, ${ }^{28}$ these data not only suggest that radial glia are cells of origin of ependymoma but also that PAX6 could be involved transforming neural progenitors into ependymoma cancer stem cells. Taken together, these data indicate that PAX6 is expressed in human ependymomas and that expression may differ between molecular and anatomic subgroups. There is a need for novel prognostic biomarkers because of the low reliability of conventional morphological grading of ependymoma. Currently, immunhistochemical data on PAX6 expression in these tumours are lacking. We, therefore, analysed the actual frequency and distribution of PAX6 protein expression in 172 human ependymal tumours, and correlate PAX6 expression with biological relevant molecular subgroups, tumour grade, anatomical location and clinical outcome.

\section{METHOD}

\section{Biological specimen}

In total, 172 available ependymoma brain tumour samples were enrolled in this retrospective study (69 female, 103 male, age range: $0-82$ years). The formalin-fixated, paraffin-embedded samples were obtained from patients undergoing surgery between 2000 and 2019 at the University Hospital Tuebingen. All samples were re-evaluated for histological diagnosis and grading according to the current WHO classification of CNS tumours. ${ }^{2}$ Tumour location, gender, survival, tumour status (primary/progression) and patient age were retrieved from the clinical records.

\section{Tissue microarray}

After microscopic evaluation, eligible representative areas of 155 tumour samples were selected and tumour cylinder probes measuring $1 \mathrm{~mm}$ in diameter were extracted from the respective area of the corresponding paraffin-embedded tumour tissue sample with a conventional tissue microarrayer (Beecher Instruments, Sun Prairie, Wisconsin, USA) and aligned as tissue microarray (TMA) on a recipient paraffin block. Subsequently, $4 \mu \mathrm{m}$ slides were cut from the TMA blocks with a microtome and dried at $80^{\circ} \mathrm{C}$ for $15 \mathrm{~min}$ and used for immunohistochemistry. Further 17 samples were evaluated on full-slides because tissue was not sufficient for transfer on TMA blocks.

\section{Immunohistochemistry and molecular analysis}

All 172 samples were previously screened with IDH1 R132H and ATRX stains as described previously to exclude potential astrocytomas. ${ }^{15}{ }^{29}$ Immunohistochemical staining of the ependymoma TMA samples and full sections was done with a Ventana BenchMark immunostainer (Ventana Medical Systems, Tucson, Arizona, USA). Pretreatment was performed with prediluted Cell Conditioning Solution CC1 $(\mathrm{pH} \mathrm{8.5)} \mathrm{for} 32$ min or $48 \mathrm{~min}$ (PAX6), followed by incubation with primary antibodies at for $32 \mathrm{~min}$ at room temperature or $37^{\circ} \mathrm{C}$ (NF-kappaB p65). Following antibodies were used: Rabbit anti human Tri-Methyl Histone K27, clone C36B11, Cell Signalling at 1:200 dilution, rabbit anti human YAP1, clone D8H1X, Abcam at 1:800, mouse anti human NF-kappaB p65, Cell Signalling at 1:8000 dilution, mouse anti human L1CAM, clone UJ127.11, Sigma, 1:8000 dilution and mouse monoclonal PAX6, clone sc81649 (Santa Cruz Biotechnology, Dallas, Texas, USA), dilution 1:200. Antibody incubation was followed by OptiView HQ Universal Linker for $12 \mathrm{~min}$, incubation with OptiView HRP Multimer for $12 \mathrm{~min}$. Both stains were finalised with counterstaining with one drop of haematoxylin for $4 \mathrm{~min}$. Each staining round was accompanied by controls using molecularly validated RELAfusion positive ependymoma for L1CAM and NFkappaB p65, breast cancer samples for YAP1, human pancreatic tissue for PAX6 (online supplemental figure 1) and glioblastoma for H3K27me3. Methylation classification data from paediatric cases was retrieved from supratentorial and PF cases enrolled in the MNP2.0, PTT2.0 or INFORM trials. ${ }^{30}$ Adult cases were classified using $\mathrm{H} 3 \mathrm{~K} 27 \mathrm{me} 3$ staining for fossa posterior tumours ${ }^{6}$ and L1CAM and NFkappaB p65 expression was used to identify RELA-fusion positive tumours. ${ }^{731}$

\section{Microscopy and statistical analysis}

TMA sections were examined by light microscopy, and photographic documentation was performed with ProgresC10 (Jenoptik, Jena). All available tissue punches from distinct regions of tumour, were analysed. L1CAM and YAP1 were evaluated for cytoplasmic expression, while nuclear staining in NFkappaB, H3K27me3, PAX6 and YAP1 counting was performed blindly with respect to all other clinical and histopathological data on tissue sections. NFkappaB was considered positive, when nuclear staining was observed in more than $5 \%$ of tumour cells. PAX6 was evalutated as nuclear staining with the help of an automated percentage assessment of digital images taken from stained slides. ImageJ software (V.1.51j8, NIH, Bethesda, Maryland, USA) together with the plugins Bio-Formats (Release V.5.4.1; Open Microscopy Environment, Madison, New Jersey, USA) and ImmunoRatio (V.1.0c, Institute of Biomedical Technology, University of Tampere, Tampere, Finland) were used. In addition to full counting, the PAX6 staining in tumours was quantified separately as follows: 0 negative (less than $1 \%$ nuclear staining), 1 (1\%-24\% tumour cells positive), 2 (25\%49\% tumour cells positive, 3 (50\%-74\% tumour cells positive) and 4 (more than $75 \%$ tumour cells positive). During the establishment of the PAX6 staining, in addition to a dilution series for optimal signal-to noise ratio, full slide sections were compared with the corresponding TMA punches in five cases to check the transferability and reproducibility of the stains. Quantitative and statistical analyses were performed using JMP V.7.0 (SAS Institute). For correlation analyses, we performed unpaired, two-tailed Student's t-test and the Fisher's exact test to identify possible significant associations or differences between two pairs. Kaplan-Meier testing was performed using a PAX6 cut-off determined by classification and regression tree (CART) analysis with progression-free survival as covariate (median follow-up time: 714 days $(95 \%$ CI mean: 936 to 1336 days, total samples: $\mathrm{n}=144$ ). Multivariate analysis using the proportional Cox hazard model for PAX6 included age and gender and tumour WHO grade. Subgroup testing was performed for grade II and III tumours combined, then again with myxopapillary ependymoma included according to the proposed future WHO classification as grade II tumours, ${ }^{13}$ for tumour separated by their anatomical compartments (supratentorial, infratentorial, spinal) and for molecular subgroups (excluding EPN-YAP and subependymomas cohorts with insufficient samples). Univariate analyses of the 
Table 1 Epidemiological details and immunohistochemistry results for ependymoma separated by molecular profile and anatomical compartment

\begin{tabular}{|c|c|c|c|c|c|c|c|c|c|}
\hline & Cases & $\begin{array}{l}\text { Primary, } \\
\text { recurrence }\end{array}$ & Mean age (range) & F/M & Tumour grades I-III & L1CAM pos & NfkappaB p65 pos & YAP1 pos & $\begin{array}{l}\text { PAX6 mean } \\
\% \text { (range) }\end{array}$ \\
\hline EPN-RELA & 10 & $5 / 5$ & $22.5(3-42)$ & $2 / 8$ & $0-0-10$ & $6 / 8$ & $8 / 8$ & $7 / 7$ & $17.1(1.9-38.3)$ \\
\hline EPN-YAP1 & 2 & $1 / 1$ & $2(1-3)$ & $1 / 1$ & $0-0-2$ & $0 / 2$ & $0 / 2$ & $1 / 2$ & $41(6.3-75.6)$ \\
\hline SE-SUP & 5 & $5 / 0$ & $50.8(40-66)$ & $2 / 3$ & $5-0-0$ & $0 / 5$ & $0 / 5$ & $4 / 5$ & $6.7(0.7-19.6)$ \\
\hline PFA & 16 & $9 / 7$ & $7.5(2-42)$ & $6 / 10$ & $0-5-11$ & $1 / 8$ & $1 / 7$ & $5 / 6$ & $32.8(1-95.6)$ \\
\hline PFB & 24 & $20 / 4$ & $45.2(20-77)$ & $11 / 13$ & $0-17-7$ & $0 / 23$ & $0 / 23$ & $22 / 23$ & $29.3(0.5-94)$ \\
\hline SE-INF & 5 & $5 / 0$ & $61.6(47-75)$ & $0 / 5$ & $5-0-0$ & $0 / 5$ & $0 / 5$ & $3 / 4$ & $3.4(0.7-10.7)$ \\
\hline MPE & 29 & $27 / 2$ & $38.4(9-60)$ & $11 / 18$ & $29-0-0$ & $0 / 27$ & $0 / 28$ & $18 / 26$ & $30.9(2.9-78.5)$ \\
\hline EPN-SPINE & 68 & $64 / 4$ & $47.1(11-82)$ & $33 / 35$ & $0-63-5$ & $2 / 65$ & $2 / 66$ & $48 / 59$ & $31(0.4-97.5)$ \\
\hline EPN-NC & 13 & $11 / 2$ & $40,61(2-74)$ & $3 / 10$ & $0-6-7$ & $0 / 12$ & $0 / 12$ & $3 / 12$ & $15(0-61.3)$ \\
\hline
\end{tabular}

EPN-NC, not classified; EPN-RELA, ependymoma RELA-fusion positive; EPN-SPINE, spinal ependymoma; EPN-YAP1, ependymoma with Yap1 fusion; MPE, myxopapillary ependymoma; PAX6, paired box 6; PFA, posterior fossa group A ; PFB, posterior fossa group B ependymoma; SE-INF, subependymoma infratentorial; SE-SUP, subependymoma supratentorial.

different variables were obtained with $95 \%$ CIs. A $p<0.05$ was considered as significant.

\section{RESULTS}

A total of 172 ependymomas (69 female, 103 male patients, mean age 39.7 years, range $0-82$ ) were included. The cohort consisted of 10 subependymoma, 29 myxopapillary ependymomas, 91 ependymoma WHO grade II and 42 anaplastic ependymoma WHO grade III. Details for each molecular group are shown in table 1.

Cytoplasmic L1CAM expression was observed in nine cases including 6/8 (75\%) ependymomas with RELA-fusion. The remaining cases were two spinal ependymomas and one positive case was a PFA ependymoma. Nuclear expression of NFkappaB was observed in $8 / 8$ (100\%) RELA-fusion positive samples, in two spinal ependymomas and in one PFA case. The three nonRELA cases positive for NFkappaB p65 were the same positive for L1CAM. YAP1 expression was not restricted to YAP-fused ependymoma and observed more or less in 111 tumours across all subgroups. The mean nuclear expression of trimethylated histone H3K27me3 in all ependymal tumours was 46\% (95\% CI $41 \%$ to $51 \%)$. As expected, a significantly reduced expression of trimethylated histone H3K27me3 was seen in PFA tumours (mean: 4\% positive nuclei) compared with highest expression levels in PFB tumours (mean: 73\% positive nuclei).

Nuclear PAX6 expression in ependymomas was highly variable, ranging from completely negative to expression in $97 \%$ tumour cells (mean: $27.5 \%, 95 \%$ CI 23.3 to $31.7, \mathrm{n}=172$, figure 1 ). PAX6 staining and expression pattern did not differ between TMA punches and full slides. 55 (32\%) samples were considered negative (PAX6 score 0). Low expression was observed in 50 samples $(29 \%$, PAX6 score 1$)$, intermediate in 30 samples $(17 \%$, PAX score 2) and high expression was seen in 18 samples (10\%, PAX6 score 3). Nineteen samples (11\%, PAX6 score 4) had very high PAX6 expression. PAX6 expression in WHO grade I and II tumours was lower compared with WHO grade III tumours (table 2). Lowest PAX6 expression levels were observed in subependymomas (mean: 5\%), while PAX6 expression in myxopapillary ependymoma (mean: 31\%) and ependymoma WHO grade II (mean: 26\%) were similar. Highest PAX6 levels were observed in anaplastic ependymoma WHO grade III (mean: 35\%). The PAX6 expression in subependymoma was significantly lower compared with myxopapillary $(\mathrm{p}=0.010)$, classical $(\mathrm{p}=0.025)$ and anaplastic ependymoma $(\mathrm{p}=0.0023)$.

There was no significant PAX6 differences between male and female $(\mathrm{p}=0.88)$ samples, between primary and recurring ependymomas $(\mathrm{p}=0.84)$ and no association of PAX6 expression with age $(p=0.182)$. Supratentorial ependymomas had significant lower PAX6 levels (mean: 15\%, p=0.012) compared with spinal cord tumours (mean 30\%). Infratentorial tumours (mean: 28\%) overlapped with supratentorial and spinal cord ependymomas.
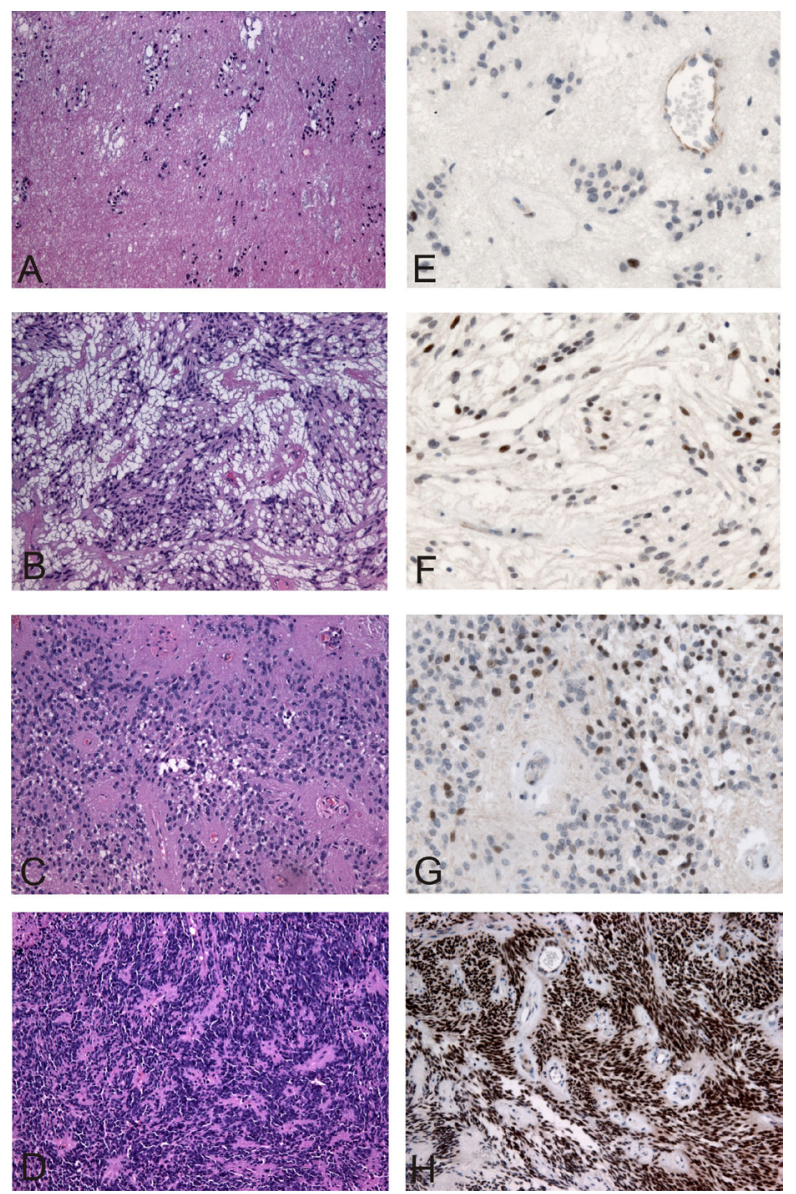

Figure 1 Histology and PAX6 immunohistochemistry results in exemplary cases. Subependymoma (A), myxopapillary ependymoma (B), WHO grade II ependymoma (C), anaplastic ependymoma (D) and their corresponding PAX6 immunohistochemical results $(\mathrm{E}-\mathrm{H})$ with nuclear staining for PAX6 (brown chromogen, (E) 1\% PAX6 positive nuclei, (F) 20\% PAX6, (G) 20\% PAX6 and (H) 96\% PAX6). PAX6, paired box 6 . 


\begin{tabular}{|c|c|c|c|}
\hline & $\mathrm{n}$ & PAX6 mean & $P$ value \\
\hline \multicolumn{4}{|l|}{ WHO Grade } \\
\hline 1 & 39 & 24.3 & \\
\hline II & 91 & 25.6 & \\
\hline III & 42 & 34.7 & 0.089 \\
\hline \multicolumn{4}{|l|}{ Histology } \\
\hline Myxopapillary & 29 & 30.9 & \\
\hline Subependymoma & 10 & 5.0 & \\
\hline Classic & 91 & 25.6 & \\
\hline Anaplastic & 42 & 34.7 & 0.0020 \\
\hline Age & 172 & & 0.182 \\
\hline \multicolumn{4}{|l|}{ Gender } \\
\hline Female & 69 & 30.7 & \\
\hline Male & 103 & 25.4 & 0.88 \\
\hline \multicolumn{4}{|l|}{ Localisation } \\
\hline Infratentorial & 45 & 28.1 & \\
\hline Spinal cord & 100 & 30.3 & \\
\hline Supratentorial & 26 & 14.8 & 0.211 \\
\hline \multicolumn{4}{|l|}{ Molecular Subgroup } \\
\hline EPN-RELA & 10 & 17.1 & \\
\hline EPN-YAP1 & 2 & 41 & \\
\hline SE-SUP & 5 & 6.7 & \\
\hline PFA & 16 & 32.8 & \\
\hline PFB & 24 & 29.3 & \\
\hline SE-INF & 5 & 3.4 & \\
\hline EPN-SPINE & 68 & 31 & \\
\hline MPE & 29 & 30.9 & \\
\hline Not classified & 13 & 15 & 0.0257 \\
\hline \multicolumn{4}{|l|}{ Tumour type } \\
\hline Primary & 147 & 27.3 & \\
\hline Recurrent & 25 & 28.4 & 0.84 \\
\hline
\end{tabular}

EPN-REAL, ependymoma RELA-fusion positive; EPN-SPINE, spinal ependymoma; EPN-YAP1, ependymoma with Yap1 fusion; MPE, Myxopapillary ependymoma; $P A X 6$, paired box 6; PFA, posterior fossa group $A ; P F B$, posterior fossa group $B ; S E-$ INF, subependymoma infratentorial; SE-SUP, subependymoma supratentorial.

PAX6 showed significant differences across molecular ependymoma subgroups (figure 2). Expression levels in YAP1-fused ependymoma (mean: 41\%) were higher compared with RELAfusion positive tumours (mean: 17\%), while PAX6 expression was similar in PF group PFA (mean: 33\%) and PFB (mean: 29\%) ependymomas. PAX6 expression in infratentorial subependymoma (mean: 3\%) was significantly lower compared with myxopapillary ependymoma (mean: $31 \%, \mathrm{p}=0.0392$ ) and to spinal ependymomas (mean: 31\%, $\mathrm{p}=0.0312$ ).

Kaplan-Meier analysis for pure histological grading in our cohort showed no significant prognostic differences between grade II WHO and grade III tumours (log-rang: $p=0.2399$, $\mathrm{n}=119$, (online supplemental figure 2A) in accordance with previous data. ${ }^{4}$ Because a recent consensus meeting has recommended future grading of myxopapillary ependymoma as grade II WHO neoplasm, ${ }^{13}$ we repeated the Kaplan-Meier analysis with MPE tumours included as WHO II tumours and obtained similar non-significant results in our cohort (Log-rang: $p=0.1243$, $\mathrm{n}=144$, (online supplemental figure $2 \mathrm{~B}$ ).

We observed a significant correlation between PAX6 expression and progression-free survival in Cox proportional hazard model (likelihood ratio: $\mathrm{p}>\chi^{2}: 0.0057, \mathrm{n}=144$, figure 3 ). CART analysis of PAX6 best fit identified an optimal PAX6 cut-off at

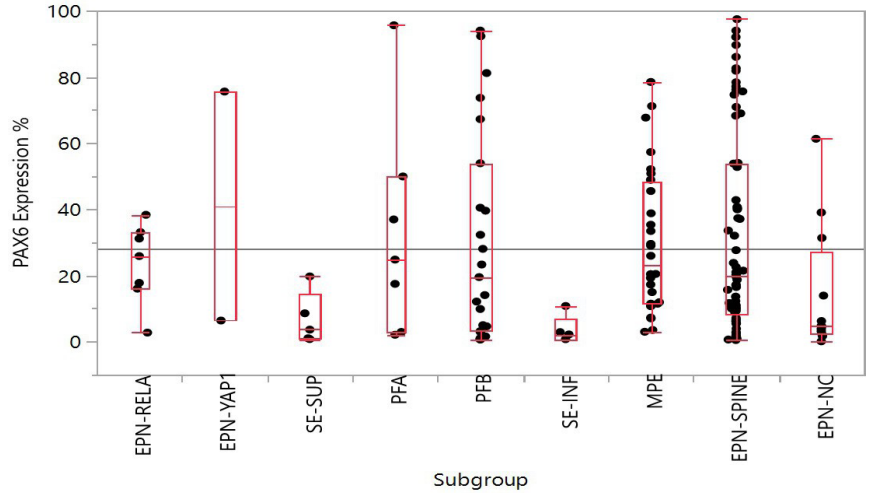

Figure 2 Box plot of distribution of PAX6 immunohistochemistry across molecular subgroups. EPN-NC, ependymoma, non-classifyable; EPN-RELA, ependymoma RELA-fusion positive; EPN-SPINE, spinal ependymoma; EPN-YAP1, ependymoma YAP1 fusion positive; MPE, myxopapillary ependymoma; PAX6, paired box 6; PFA, ependymoma posterior fossa group $A ; P F B$ : ependymoma posterior fossa group B, SE-INF, subependymoma infratentorial; SE-SUP, subependymoma supratentorial.

19.45\% (online supplemental figure 3). Using this cut-off, PAX6 expression $\geq 19.45 \%$ was observed in 68 ependymomas (median progression-free survival: 1526 days, and expression $<19.45 \%$ was observed in 76 tumours (median progression-free survival: 473 days, log-rang: $p<0.0001$, figure 4 ). Because the cut-off of $19.45 \%$ is very specific, we repeated Kaplan-Maier analysis with slightly different thresholds for practical purposes in PAX6 immunohistochemistry scoring and results remained significant using the threshold values (cut-off 17\%: log-rang $\mathrm{p}=0.0003$; cut-off 20\%: $p=0.0002$; cut-off 22\%: $p=0.0001$ ).

Due to their prognostic role, we next performed Kaplan-Meier analysis for the relevant anatomic compartments separately. In infratentorial ependymomas, a significant better outcome for PAX6 at or above the cut-off (Log-rang $\mathrm{p}<0.049, \mathrm{n}=17$, median time: 1488 days) was observed compared with ependymomas with PAX6 <19.45 ( $n=20$, median time: 638 days). In spinal cord ependymomas outcome in PAX6 $\geq 19.45 \%$ was significantly better ( $\mathrm{p}<0.0001, \mathrm{n}=45$, median: 1225 days) compared with tumours with PAX6 <19.45 ( $\mathrm{n}=44,258$ days). Due to low numbers, in supratentorial tumours no significant better outcome was seen in in PAX6 $\geq 19.45 \%$ tumours ( $p=0.87, n=6$, 2162 days) compared with PAX6 <19.45 ( $\mathrm{n}=14,1198$ days).

A similar prognostic role for PAX6 was observed in ependymomas separated by their molecular profile (figure 4, table 3). A significant better outcome for PAX6 at or above the cut-off 19.45 was observed in RELA-fusion positive ependymoma $(\mathrm{p}=0.0136$, table 3$)$, in posterior group $B$ ependymomas $(p=0.0341)$ and in spinal ependymoma $(p=0.0001$, figure 3$)$. Due to the low numbers of samples in some subgroups, survival analysis could not evaluated in all molecular cohorts.

\section{DISCUSSION}

Ependymal tumours, often histologically identical, comprise genetically distinct subgroups associated with distinct anatomic compartments and imprinted methylation signature inherited from their cells of origin. ${ }^{5}$ Similar to radial glia cells in the corresponding region of the CNS, Pax 6 expression has been reported in animal ependymoma models, ${ }^{27} 28$ but data on PAX6 protein expression was lacking in human ependymal tumours. We, therefore, conducted an immunohistochemical study of 


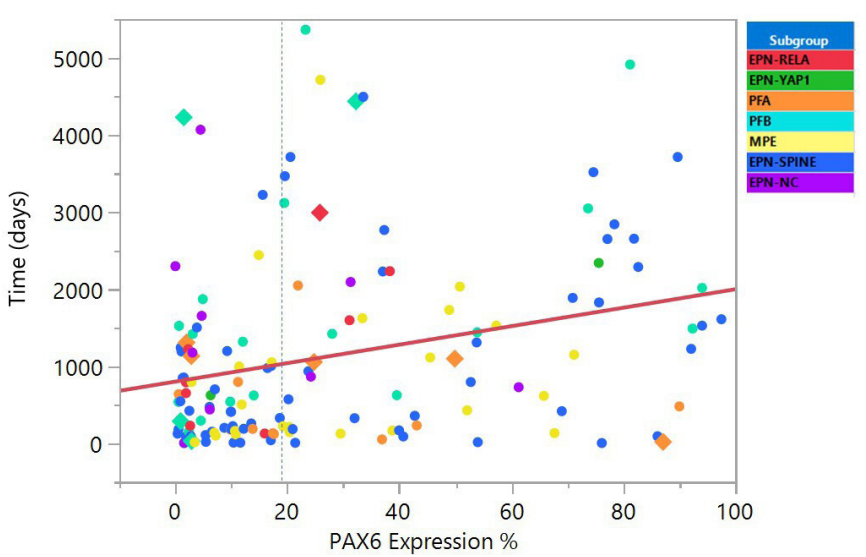

Figure 3 Scatterplot of PAX6 staining percentage plotted against progression-free survival with molecular subtypes marked by colour of points. The blue dotted line marks the $19.45 \%$ cut-off determined by Classification and Regression Tree, the red line marks linear fit of correlation ( $p=0.0022$ ) which remains significant in multivariate analysis (Proportional hazard fit, likelihood ratio: $p=0.0057$ ), censored values are shown as squares. EPN-NC, ependymoma, non-classifyable; EPN-RELA, ependymoma RELA-fusion positive; EPN-SPINE, spinal ependymoma; EPN-YAP1, ependymoma with Yap1 fusion; MPE, Myxopapillary ependymoma; PAX6, paired box 6; PFA, posterior fossa group A; PFB, posterior fossa group $B$.

PAX6 in 172 ependymal tumours of different anatomic components and molecular backgrounds and correlated its expression to histology, WHO grade, anatomical location and molecular subgroups. Consistent with the putative cell of origin of ependymal tumours, we identified PAX6-positive tumour cells in the majority (68\%) of human ependymoma and PAX6 expression in our cohort was not significantly altered after treatment at recurrence. Also consistent with its putative role as transcription factor, nuclear PAX6 was quite variable and showed a trend to increased expression with tumour grading (table 2). Similarly, in a study examining 111 ductal breast cancer samples, approximately two-thirds $(n=75)$ of the tumours showed low number of PAX6 positive tumour cells. ${ }^{32}$ This contrasts with data in
Table 3 Kaplan Meier progression-free survival (PFS) data for molecular groups separated by PAX6 cut-off 19.45

\begin{tabular}{|c|c|c|c|c|c|}
\hline $\begin{array}{l}\text { Molecular } \\
\text { subgroup }\end{array}$ & $\begin{array}{l}\text { n PAX6 } \\
<19.45\end{array}$ & $\begin{array}{l}\text { Median } \\
\text { PFS (d) } \\
\text { PAX6 } \\
<19.45\end{array}$ & $\begin{array}{l}\text { n PAX6 } \\
\geq 19.45\end{array}$ & $\begin{array}{l}\text { Median PFS (d) } \\
P A X 6 \geq 19.45\end{array}$ & $\begin{array}{l}\text { Log-rang } \\
P \text { value }\end{array}$ \\
\hline EPN-RELA & 5 & 651 & 3 & 2232 & $0.0136^{*}$ \\
\hline PFA & 8 & 412 & 7 & 1262 & 0.4323 \\
\hline PFB & 12 & 1318 & 10 & 2530 & $0.0341^{*}$ \\
\hline EPN-SPINE & 33 & 306 & 30 & 1417 & $0.0001^{*}$ \\
\hline MPE & 11 & 222 & 14 & 864 & 0.1778 \\
\hline
\end{tabular}

*Significant.

EPN-RELA, ependymoma RELA-fusion positive; EPN-SPINE, spinal ependymoma; MPE, Myxopapillary ependymoma; PAX6, paired box 6; PFA, posterior fossa group $A ;$ PFB, posterior fossa group $B$.

pancreatic cancer where PAX6 in the majority of tumours stained more than $50 \%$ of tumour nuclei. ${ }^{33}$ Although PAX6 was widely distributed up to $97.5 \%$ positive tumour nuclei in one case, the mean nuclear PAX6 expression in ependymoma was $27.5 \%$ and differed significantly between supratentorial ependymomas $(15 \%)$ and spinal cord tumours (30\%), indicating that PAX6 is heterogeneously expressed in a subset of tumour cells. In nonneoplastic human brain, PAX6 is expressed in the cerebellum. ${ }^{15}$ In accordance with this data, PAX6 expression in infratentorial ependymomas was higher compared with supratentorial tumours (table 2). Data from previous studies showed that infratentorial ependymomas derive from regionally specific stem cells bearing a radial glial cell phenotype. ${ }^{34}$ Transcriptome analysis showed the radial glial neural stem cell marker PAX6 to be expressed at a higher level in ependymomas compared with glioblastomas. ${ }^{5}$ PAX6 is also expressed in subsets of interneurons in the ventral spinal cord in mice. ${ }^{35}$ Interestingly in our cohort, PAX6 in spinal ependymomas was higher and clearly associated with the classical ependymoma histology, thus, clearly segregating subependymomas with overall low PAX6 expression from all other ependymal tumours. Our immunohistochemical data matches transcriptome results indicating lower PAX6 levels in subependymoma. ${ }^{5}$ Some authors believe that subependymal tumours
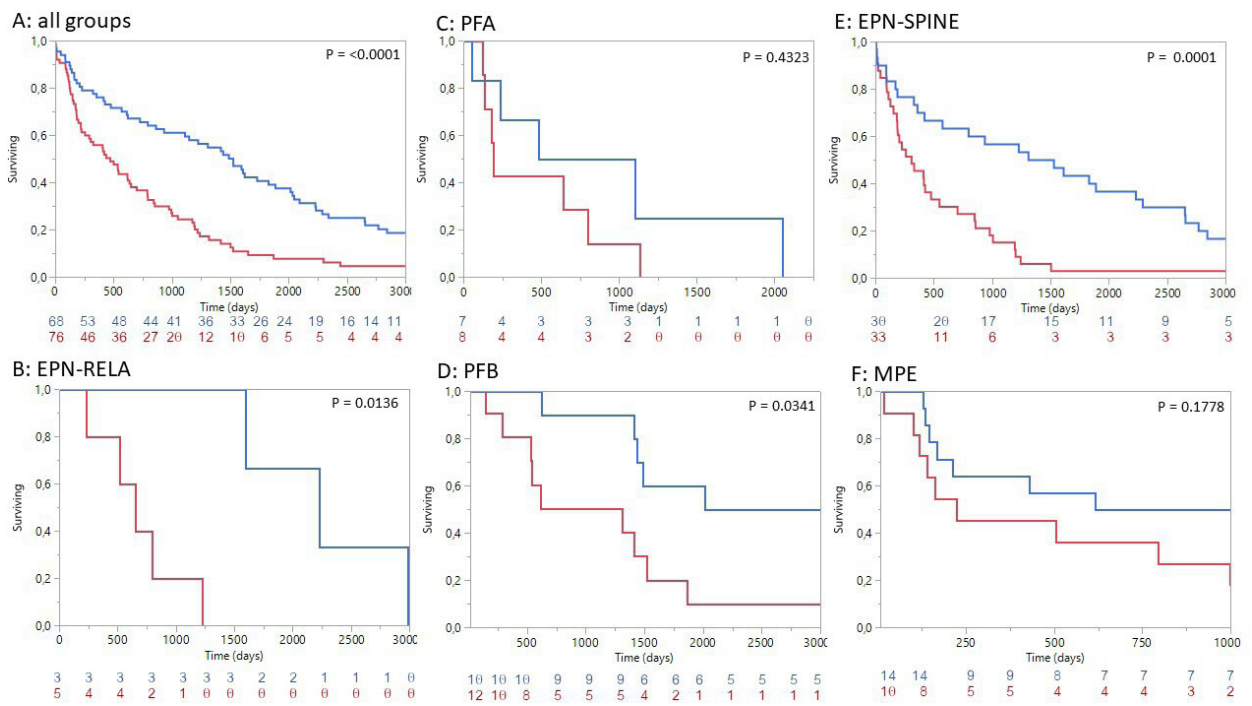

Figure 4 Kaplan-Meier analysis for ependymomas separated by PAX6 expression in molecular subgroupsblue: $P A X 6 \geq 19.45$ vs red: $P A X 6<19.45$, for details see table 3. EPN-RELA, ependymoma RELA-fusion positive; EPN-SPINE, spinal Ependymoma; MPE, Myxopapillary ependymoma; PAX6, paired box 6; PFA, ependymoma posterior fossa group A; PFB, ependymoma posterior fossa group $B$. 
originate from a mixture of astrocytes of the subependymal plate and ependymal cells rather than of pure ependymal origin. ${ }^{36}$ Clinically, most of these tumours are inactive and show a very favourable prognosis, again differing from the course seen in other ependymal tumours. Even WHO grade I myxopapillary ependymomas recur more frequently, so that some authors propose WHO grade II for these tumours to differentiate them from indolent subependymomas. ${ }^{13}$ Our observation that PAX6 levels in myxopapillary ependyomas were similar to grade II ependymomas fits to this notion. Our data also hints at a prognostic role of PAX6 in WHO grade II and III ependymoma with a better outcome in cases with PAX6 expression levels above the cut-off determined in CART analysis. A higher expression of PAX6 was observed in anaplastic ependymoma. This observation further supports the low reliability of conventional morphological grading in WHO II and III ependymoma and stresses the need for novel prognostic markers. The significant role for PAX6 at or above the cut-off 19.45 was retained when we separated our tumours by their prognostic relevant anatomic compartments. Furthermore beside a significant role in supratentorial, infratentorial and spinal cord ependymomas, we also found PAX6 to be prognostic relevant in RELA-fusion positive ependymoma and in posterior group B ependymomas (table 3). The determined cut-off $19.45 \%$ in our cohort is close to the PAX6 median $18.9 \%$. Because there are no substantial differences for slightly different threshold values (such as cut-offs at 17\%, 20\% or $22 \%$ ), a simplified approach of $20 \%$ for practical purposes may be used in future PAX6 immunohistochemistry scoring. Of course, our observations are limited due to the retrospective nature of our study and the divergent treatment depending on WHO grades and low numbers of cases, especially of YAP1fused cases. The prognostic potential should be verified in an independent validation cohort. In general, low PAX6 expression in our combined cohort of 144 tumours was associated with poor outcome. Similar observations have been made in other cancer types. Kiselev et al reported in node positive small cell lung cancer for patients with high PAX6 expression a median survival of 127.4 months, vs 22.9 months for patients with low PAX6 expression. ${ }^{17}$ Another study identified down-regulation of PAX6 by promoter methylation was associated with poor prognosis in 143 non-small cell lung cancer specimen. ${ }^{37}$ Consistent with these results, PAX6 overexpression through transfection in glioma cell lines suppresses glioma growth and the survival of mice with tumours implanted was prolonged. ${ }^{20}$ It is noteworthy that supratentorial ependymomas frequently harbour chromothripsis involving Chr.11q13 and extending further across Chr.11, ${ }^{7}$ but deletions of Chr. 11p13 involving PAX6 is usually

\section{Take home messages}

- Paired box 6 (PAX6) is expression is present in $68 \%$ of ependymal tumours and is significantly higher in spinal cord tumours compared with supratentorial tumours.

- Nuclear expression of PAX6 is highly variable and is significantly lower in subependymoma compared with other histological variants.

- PAX6 shows significant differences across molecular ependymoma subgroups with lower expression levels in RELA-fusion positive tumours compared with posterior fossa group $A$ and $B$ tumours.

- There is a significant correlation between PAX6 expression level and progression-free survival. not observed in ependymomas. The modulating factor for PAX6 expression in ependymomas and if there is an association with PAX6's role as tumour suppressor remains to be identified. Taken together, we demonstrate that PAX6 is frequently expressed in human ependymal tumours and immunohistochemistry may be helpful in determining prognostic relevant subgroups.

\section{Author affiliations}

${ }^{1}$ Department of Neuropathology, Institute of Pathology and Neuropathology, University Hospital of Tuebingen, Eberhard Karls University of Tuebingen, Tuebingen, Germany

${ }^{2}$ Department of Neurosurgery, Eberhard Karls University of Tuebingen, BadenWürttemberg, Germany

${ }^{3}$ Center for Neuro-Oncology, Comprehensive Cancer Center Tuebingen-Stuttgart, University Hospital of Tuebingen, Eberhard Karls University of Tuebingen, Tuebingen, Germany

${ }^{4}$ Division of Pediatric Neurosurgery, Department of Neurosurgery, University Hospital of Tuebingen, Eberhard Karls University of Tuebingen, Tuebingen, Germany ${ }^{5}$ Department Pediatric Hematology/Oncology, Children's University Hospital, Eberhard Karls University of Tuebingen, Tuebingen, Germany

${ }^{6}$ Interdisciplinary Division of Neuro-Oncology, University Hospital of Tuebingen, Eberhard Karls University of Tuebingen, Tuebingen, Germany

\section{Handling editor Runjan Chetty.}

Acknowledgements We like to thank Manuel Gödan for assistance with immunohistochemistry.

Contributors All authors made substantial contributions to the study, that is, conception, design, sample acquisition, clinical data acquisition, data analysis, data interpretation and coordination of experiments and collaborations. JT and JS designed and coordinated the study, evaluated, analysed and interpreted the results. JS wrote the first draft of the manuscript, created figures and data table. JH, MS, MR and ME provided clinical data. All authors read, modified the manuscript and approved the final version.

Funding The authors have not declared a specific grant for this research from any funding agency in the public, commercial or not-for-profit sectors.

Competing interests None declared.

Patient consent for publication Not required.

Ethics approval The study was authorised by the University ethics board (number 057/2018B02).

Provenance and peer review Not commissioned; externally peer reviewed.

Data availability statement Data are available on reasonable request. All data relevant to the study are included in the article or uploaded as online supplemental information. The individual datasets generated during and/or analysed during the current study are not publicly available due to privacy of research participants but are available from the corresponding author on reasonable request.

Supplemental material This content has been supplied by the author(s). It has not been vetted by BMJ Publishing Group Limited (BMJ) and may not have been peer-reviewed. Any opinions or recommendations discussed are solely those of the author(s) and are not endorsed by BMJ. BMJ disclaims all liability and responsibility arising from any reliance placed on the content. Where the content includes any translated material, BMJ does not warrant the accuracy and reliability of the translations (including but not limited to local regulations, clinical guidelines, terminology, drug names and drug dosages), and is not responsible for any error and/or omissions arising from translation and adaptation or otherwise.

Open access This is an open access article distributed in accordance with the Creative Commons Attribution Non Commercial (CC BY-NC 4.0) license, which permits others to distribute, remix, adapt, build upon this work non-commercially, and license their derivative works on different terms, provided the original work is properly cited, appropriate credit is given, any changes made indicated, and the use is non-commercial. See: http://creativecommons.org/licenses/by-nc/4.0/.

\section{ORCID iD}

Jens Schittenhelm http://orcid.org/0000-0002-9168-6209

\section{REFERENCES}

1 Mack SC, Taylor MD. The genetic and epigenetic basis of ependymoma. Childs Nerv Syst 2009;25:1195-201.

2 Wesseling P, Capper D. WHO 2016 Classification of gliomas. Neuropathol Appl Neurobiol 2018:44:139-50.

3 Godfraind C. Classification and controversies in pathology of ependymomas. Childs Nerv Syst 2009;25:1185-93. 
4 Tihan T, Zhou T, Holmes E, et al. The prognostic value of histological grading of posterior fossa ependymomas in children: a children's Oncology Group study and a review of prognostic factors. Mod Pathol 2008;21:165-77.

5 Paitler KW, Witt H, Sill M, et al. Molecular classification of ependymal tumors across all CNS compartments, histopathological grades, and age groups. Cancer Cell 2015;27:728-43.

6 Panwalkar P, Clark J, Ramaswamy V, et al. Immunohistochemical analysis of H3K27me3 demonstrates global reduction in group-A childhood posterior fossa ependymoma and is a powerful predictor of outcome. Acta Neuropathol 2017;134:705-14.

7 Parker M, Mohankumar KM, Punchihewa C, et al. C11 orf95-RELA fusions drive oncogenic NF- $\mathrm{KB}$ signalling in ependymoma. Nature 2014;506:451-5.

8 Zschernack V, Jünger ST, Mynarek M, et al. Supratentorial ependymoma in childhood: more than just RelA or YAP. Acta Neuropathol 2021;141:455-66.

9 Zheng T, Ghasemi DR, Okonechnikov K, et al. Cross-Species genomics reveals oncogenic dependencies in ZFTA/C11 orf95 fusion-positive supratentorial ependymomas. Cancer Discov 2021. doi:10.1158/2159-8290.CD-20-0963. [Epub ahead of print: 20 Apr 2021].

10 Andreiuolo F, Varlet P, Tauziède-Espariat A, et al. Childhood supratentorial ependymomas with YAP1-MAMLD1 fusion: an entity with characteristic clinical, radiological, cytogenetic and histopathological features. Brain Pathol 2019;29:205-16.

11 Ebert C, von Haken M, Meyer-Puttlitz B, et al. Molecular genetic analysis of ependymal tumors. NF2 mutations and chromosome 22q loss occur preferentially in intramedullary spinal ependymomas. Am J Pathol 1999;155:627-32.

12 Ghasemi DR, Sill M, Okonechnikov K, et al. MYCN amplification drives an aggressive form of spinal ependymoma. Acta Neuropathol 2019:138:1075-89.

13 Ellison DW, Aldape KD, Capper D, et al. cIMPACT-NOW update 7: advancing the molecular classification of ependymal tumors. Brain Pathol 2020;30:863-6.

14 Simpson TI, Price DJ. Pax6; a pleiotropic player in development. Bioessays 2002:24:1041-51

15 Pibiri V, Ravarino A, Gerosa C, et al. Stem/Progenitor cells in the developing human cerebellum: an immunohistochemical study. Eur J Histochem 2016;60:2686.

16 Lang D, Powell SK, Plummer RS, et al. Pax genes: roles in development, pathophysiology, and cancer. Biochem Pharmacol 2007;73:1-14.

17 Kiselev Y, Andersen S, Johannessen C, et al. Transcription factor Pax6 as a nove prognostic factor and putative tumour suppressor in non-small cell lung cancer. Sci Rep 2018;8:5059.

18 Muratovska A, Zhou C, He S, et al. Paired-Box genes are frequently expressed in cancer and often required for cancer cell survival. Oncogene 2003;22:7989-97.

19 Mascarenhas JB, Young KP, Littlejohn EL, et al. Pax6 is expressed in pancreatic cancer and actively participates in cancer progression through activation of the Met tyrosine kinase receptor gene. J Biol Chem 2009;284:27524-32.
20 Zhou Y-H, Tan F, Hess KR, et al. The expression of Pax6, PTEN, vascular endothelial growth factor, and epidermal growth factor receptor in gliomas: relationship to tumor grade and survival. Clin Cancer Res 2003;9:3369-75.

21 Chang JY, Hu Y, Siegel E, et al. Pax6 increases glioma cell susceptibility to detachment and oxidative stress. J Neurooncol 2007;84:9-19.

22 Pinto GR, Clara CA, Santos MJ, et al. Mutation analysis of gene Pax6 in human gliomas. Genet Mol Res 2007;6:1019-25.

23 Huang B-S, Luo Q-Z, Han Y, et al. MiR-223/PAX6 axis regulates glioblastoma stem cell proliferation and the chemo resistance to TMZ via regulating PI3K/Akt pathway. I Cell Biochem 2017;118:3452-61.

24 Al-Awadhi FH, Salvador-Reyes LA, Elsadek LA, et al. Largazole is a brain-penetrant class I HDAC inhibitor with extended applicability to glioblastoma and CNS diseases. ACS Chem Neurosci 2020;11:1937-43.

25 Mack SC, Pajtler KW, Chavez L, et al. Therapeutic targeting of ependymoma as informed by oncogenic enhancer profiling. Nature 2018:553:101-5.

26 Pajtler KW, Wei Y, Okonechnikov K, et al. Yap1 subgroup supratentorial ependymoma requires TeaD and nuclear factor I-mediated transcriptional programmes for tumorigenesis. Nat Commun 2019;10:3914.

27 Poppleton H, Gilbertson RJ. Stem cells of ependymoma. Br J Cancer 2007:96:6-10.

28 Heins N, Malatesta P, Cecconi F, et al. Glial cells generate neurons: the role of the transcription factor Pax6. Nat Neurosci 2002;5:308-15.

29 Ebrahimi A, Skardelly M, Bonzheim I, et al. Atrx immunostaining predicts IDH and H3F3A status in gliomas. Acta Neuropathol Commun 2016;4:60.

30 Selt F, Deiß A, Korshunov A, et al. Pediatric targeted therapy: clinical feasibility of personalized diagnostics in children with relapsed and progressive tumors. Brain Pathol 2016;26:506-16.

31 Pietsch T, Wohlers I, Goschzik T, et al. Supratentorial ependymomas of childhood carry C11 orf95-RELA fusions leading to pathological activation of the NF- $\mathrm{KB}$ signaling pathway. Acta Neuropathol 2014;127:609-11.

32 Xia $X$, Yin W, Zhang $X$, et al. Pax6 overexpression is associated with the poor prognosis of invasive ductal breast cancer. Oncol Lett 2015;10:1501-6.

33 Lai J-P, Mertens RB, Mirocha J, et al. Comparison of Pax6 and Pax8 as immunohistochemical markers for pancreatic neuroendocrine tumors. Endocr Pathol 2015;26:54-62.

34 Taylor MD, Poppleton $\mathrm{H}$, Fuller C, et al. Radial glia cells are candidate stem cells of ependymoma. Cancer Cell 2005;8:323-35.

35 Panayiotou E, Panayi E, Lapathitis G, et al. Pax6 is expressed in subsets of V0 and V2 interneurons in the ventral spinal cord in mice. Gene Expr Patterns 2013;13:328-34.

36 Fu YS, Chen AT, Kay S, et al. Is subependymoma (subependymal glomerate astrocytoma) an astrocytoma or ependymoma? A comparative ultrastructural and tissue culture study. Cancer 1974;34:1992-2008.

37 Zhang X, Yang X, Wang J, et al. Down-Regulation of Pax 6 by promoter methylation is associated with poor prognosis in non small cell lung cancer. Int I Clin Exp Pathol 2015;8:11452-7. 